\title{
Anesthetic Techniques Influence the Induction of Pulmonary Capillary Hemorrhage During Diagnostic Ultrasound Scanning in Rats
}

Douglas L. Miller, PhD, Chunyan Dou, MD, Krishnan Raghavendran, MD

Received February 24, 2014, from the Departments of Radiology (D.L.M., C.D.) and Surgery (K.R.), University of Michigan Health System, Ann Arbor, Michigan USA. Revision requested April 7, 2014. Revised manuscript accepted for publication May 23, 2014.

This study was supported by the National Heart Lung and Blood Institute via grant HL116434.

Address correspondence to Douglas L. Miller, $\mathrm{PhD}$, Department of Radiology, University of Michigan Health System, 3240AMedical Sciences Building I, 1301 Catherine St, Ann Arbor, MI 48109-5667 USA.

E-mail:douglm@umich.edu

\section{Abbreviations}

ANOVA, analysis of variance; bpm, beats per minute; $M I$, mechanical index; $S P_{\mathrm{O} 2}$, peripheral capillary oxygen saturation; US, ultrasound

doi:10.7863/ultra.34.2.289
Objectives - Pulmonary capillary hemorrhage can be induced by diagnostic ultrasound (US) during direct pulmonary US scanning in rats. The influence of specific anesthetic techniques on this bioeffect was examined.

Methods - Ketamine plus xylazine has been used previously. In this study, the influence of intraperitoneal injections of ketamine and pentobarbital, inhalational isoflurane, and the supplemental use of xylazine with ketamine and isoflurane was tested. A diagnostic US machine with a 7.6-MHz linear array was used to image the right lung of anesthetized rats in a warmed water bath at different mechanical index (MI) settings. Pulmonary capillary hemorrhage was assessed by measuring comet tail artifacts in the image and by morphometry of the hemorrhagic areas on excised lungs.

Results - Pulmonary capillary hemorrhage was greatest for pentobarbital, lower for inhalational isoflurane, and lowest for ketamine anesthesia, with occurrence thresholds at MIs of about $0.44,0.8$, and 0.8 , respectively. Addition of xylazine produced a substantial increase in hemorrhage and a significant proportion of hemorrhage occurrence for ketamine at an $\mathrm{MI}$ of $0.7(P<.01)$ and for isoflurane at an $\mathrm{MI}$ of $0.52(P<.01)$.

Conclusions - Ketamine plus xylazine and pentobarbital yield lower thresholds than ketamine or isoflurane alone by nearly a factor of 2 in MI. These results suggest that the choice of the anesthetic agent substantially modifies the relative risks of pulmonary capillary hemorrhage from pulmonary US.

Key Words - anesthetic techniques; comet tail artifact; point-of-care ultrasound; pulmonary hemorrhage; pulmonary ultrasound; ultrasound bioeffects

$\mathrm{P}$ ulsed ultrasound (US) can induce pulmonary capillary hemorrhage in mammals, which raises some concern for diagnostic US in medicine. This bioeffect was discovered more than 20 years ago ${ }^{1}$ and was evaluated in subsequent research for dependence on various pulsed US parameters to assess the possible risk for diagnostic US. Reported exposure thresholds for pulmonary capillary hemorrhage found in studies of the response to varied peak rarefactional pressure amplitude were reviewed by the American Institute of Ultrasound in Medicine. ${ }^{2}$ The results were interpreted in terms of the mechanical index (MI), which is an exposure index displayed on the screens of most US machines. The MI concept can be used for research by adjusting the peak rarefactional pressure amplitude measured in water for attenuation and dividing by the square root of the pulse center frequency. The thresholds, which were obtained mostly in anesthetized mice, corresponded 
roughly to an MI value of $0.63 .^{2}$ For reference, the guideline upper limit for diagnostic US is an MI of 1.9. ${ }^{3}$ Ultrasound-induced pulmonary capillary hemorrhage was found at about the same derated peak rarefactional pressure amplitudes in mice, rats, rabbits, pigs, and monkeys. A meta-analysis of 14 studies in mice and rats found that the pulmonary capillary hemorrhage effect had widely varying thresholds and appeared to depend on physical parameters, including pulse duration, pulse repetition frequency, and exposure duration, in addition to peak rarefactional pressure amplitude and frequency. ${ }^{4}$ In another authoritative review by the American Institute of Ultrasound in Medicine, patient pulmonary US exposure was expected to occur during incidental exposure to echocardiography, which was not thought to pose a consistently defined risk. ${ }^{5}$ However, this overview of the pulmonary capillary hemorrhage problem has changed in recent years for two reasons.

First, most of the available data were obtained with pulsed US from laboratory exposure systems, and the applicability to actual diagnostic US was uncertain. Diagnostic US was used for studies in monkeys ${ }^{6}$ and rats, ${ }^{7}$ but the results were mixed. A study of human lungs after echocardiography indicated that $3.5-\mathrm{MHz}$ diagnostic US up to $2.4 \mathrm{MPa}$ $(\mathrm{MI}=1.3)$, which was thought to interact with the lung as incidental exposure, did not cause pulmonary capillary hemorrhage. ${ }^{8}$ In a recent study in rats from our laboratory, diagnostic US from a 7.6-MHz linear array was used to directly scan the right lung of anesthetized rats in a warmed water bath. ${ }^{9}$ The image displayed growing comet tail artifacts, which were indicative of pulmonary capillary hemorrhage on the surface of the lung. Pulmonary capillary hemorrhage was observed for several groups of rats scanned at a range of MI settings for 5 minutes, and a threshold for hemorrhage occurrence was indicated for an $\mathrm{MI}$ of about 0.44 . This result appears to be lower than expected from the meta-analysis of physical parameters. ${ }^{4}$ The etiology of pulmonary capillary hemorrhage induced by diagnostic US remains poorly understood.

Second, direct pulmonary US examination has become a valuable diagnostic aid for pneumonia, pulmonary edema and effusion, pulmonary embolism, atelectasis, diffuse parenchymal disease, adult and newborn respiratory distress syndrome, and lung cancer. ${ }^{10}$ Diagnostic US imaging has largely replaced chest radiography for pleural effusion, pneumothorax, hemothorax, and posttraumatic lung contusion. ${ }^{11,12}$ Pulmonary US is rapidly becoming routine in intensive care, ${ }^{13-15}$ emergency care, ${ }^{16}$ and other point-ofcare settings. ${ }^{17}$ This adoption of routine pulmonary US completely changes the diagnostic US-induced pulmonary capillary hemorrhage problem of incidental exposure to one of direct, deliberate examination. The problem requires further examination from new perspectives to clarify potential patient risks and provide guidance for the safe use of pulmonary US.

One aspect of the problem in need of elaboration is the role of biological and physiologic factors. Age is one biological factor that has been explored. For mice and rats, pulmonary capillary hemorrhage thresholds appeared to have little dependence on age. ${ }^{18,19}$ Neonatal swine also had pulmonary capillary hemorrhage thresholds comparable to those of adult mice. ${ }^{20}$ For older pigs, the experimentation is more difficult due to the size of the animals, but this animal model more closely simulates humans. O'Brien et $\mathrm{al}^{21}$ found that older pigs were more sensitive than neonatal or middleaged pigs to 3.1-MHz pulsed US for 10-second exposures.

Other physiologic conditions may also be important. One key factor for research is the use of anesthetics. For general anesthesia in mice and rats, intraperitoneal injection of ketamine plus xylazine is typically used; for example, studies of rats and mice reviewed by Church and O'Brien ${ }^{4}$ all used ketamine plus xylazine. The doses were $87 \mathrm{mg} / \mathrm{kg}$ ketamine plus $13 \mathrm{mg} / \mathrm{kg}$ xylazine in rats or mice, ${ }^{21-24} 100 \mathrm{mg} / \mathrm{kg}$ ketamine plus $10 \mathrm{mg} / \mathrm{kg}$ xylazine in rats, ${ }^{7}$ and 200 $\mathrm{mg} / \mathrm{kg}$ ketamine plus $10 \mathrm{mg} / \mathrm{kg}$ xylazine in mice. ${ }^{1,17,25,26}$ The presently recommended anesthetic doses of $91 \mathrm{mg} / \mathrm{kg}$ ketamine and $9 \mathrm{mg} / \mathrm{kg}$ xylazine were used in our previous study. ${ }^{9}$

Ketamine is a dissociative anesthetic, which immobilizes with little respiratory depression, whereas xylazine is a sedative and analgesic, which produces substantial cardiovascular and respiratory depression. ${ }^{27}$ Xylazine has been reported to induce pulmonary edema in rats at relatively high doses of 21 to $45 \mathrm{mg} / \mathrm{kg}^{28,29} \mathrm{In}$ a previous study using bronchoalveolar lavage albumin levels as representative of permeability injury, the normal doses of xylazine used for anesthesia did not produce a significant injury when compared with ketamine alone. However, the omission of xylazine reduced the size of the comet tail artifacts produced during US scanning and significantly reduced the erythrocyte count in the bronchoalveolar lavage fluid found after scanning. ${ }^{30}$ Statistical analysis using analysis of variance (ANOVA) suggested that a synergistic interaction occurred between the US scanning and the use of xylazine.

The goal of this study was to examine the influence of commonly used anesthetics and drug combinations on the induction of pulmonary capillary hemorrhage by diagnostic US scanning, using our previous methods. ${ }^{9}$ A common anesthetic for rodents is pentobarbital. This barbiturate yields prolonged anesthesia and can cause cardiovascular and respiratory system depression. ${ }^{27}$ Inhalation anesthesia 
is typically used for larger animals but is also valuable for short-term anesthesia in rodents. Isoflurane typically is applied at $1 \%$ to $5 \%$ in oxygen or air from a vaporizing machine. Thresholds were determined using ketamine without xylazine, pentobarbital, and isoflurane. Pulmonary capillary hemorrhage results for ketamine with and without xylazine were compared. In addition, the effect of the addition of xylazine to isoflurane was tested. The results show substantial influences of the specific anesthetics on USinduced pulmonary capillary hemorrhage.

\section{Materials and Methods}

\section{Animal Preparation}

All in vivo animal procedures were conducted with the approval and guidance of the University Committee on Use and Care of Animals. Female Sprague Dawley rats (CD IGS strain; Charles River Laboratories, Wilmington, MA) were used for this research. The study progressed in 3 parts involving ketamine, pentobarbital, or isoflurane anesthesia.

For the ketamine anesthesia, 36 rats weighing $224 \pm$ $15 \mathrm{~g}$ (mean $\pm \mathrm{SD})$ were used, with 2 animals dying shortly after administration of the agent. There were 6 scan groups of 5 rats each and a sham group of 4 rats. The ketamine (Ketaved ketamine hydrochloride injection; Vedco, Inc, St Joseph, MO) was injected to deliver $100 \mathrm{mg} / \mathrm{kg}$ intraperitoneally for 3 scan groups and the sham group. In the additional 3 scan groups, ketamine 91 at $\mathrm{mg} / \mathrm{kg}$ intraperitoneally was supplemented with $9 \mathrm{mg} / \mathrm{kg}$ intraperitoneal xylazine (AnaSed xylazine injection; Akorn, Inc, Decatur, IL), given either before the US scanning or immediately after scanning, representing a control for the potential effects of xylazine that are not dependent on scanning.

For pentobarbital anesthesia, 31 rats weighing $242 \pm 14$ $\mathrm{g}$ were used, with 1 animal excluded for technical problems. There were 6 groups of 5 rats each, including 5 scan groups and 1 sham group. Pentobarbital (Nembutal sodium solution; Akorn, Inc) was injected at a dose of $50 \mathrm{mg} / \mathrm{kg}$ intraperitoneally.

Inhalational use of isoflurane produces moderate cardiovascular and respiratory depression but can be rapidly modulated to yield the required level of anesthesia. ${ }^{26}$ Oxygen was tried as the carrier gas in preliminary testing; however, this method seemed to produce spontaneous comet tail artifacts and areas of atelectasis on some rat's lungs. The use of $100 \%$ oxygen can lead to problems with pulmonary function due to absorptive atelectasis, a serious potential problem with inhalational anesthesia. ${ }^{31}$ This problem can be mitigated by use of lower oxygen con- centrations or air (ie, $20 \%$ oxygen). For this study, air was the isoflurane vehicle. Thirty rats weighing $227 \pm 10 \mathrm{~g}$ were assigned to 6 scan groups with air as the carrier gas, mitigating the problems found with oxygen. The isoflurane (VETone isoflurane inhalation anesthetic; MWI VetOne, Meridian, ID) was delivered by a vaporizer (Surgivet Isotec 4; Smiths Medical ASD, Inc, St Paul, MN) at a concentration of $5 \%$ for induction. The concentration was subsequently reduced to as low as $1 \%$ for maintenance during handling and scanning. For 2 groups, xylazine was injected at anesthesia (before scanning) to assess the influence of this sedative on pulmonary capillary hemorrhage.

For all animals, the right thorax was shaved after anesthesia and depilated to allow good US transmission into the body. The rats were mounted in dorsal recumbency on a plastic board, which then was mounted vertically in a $38^{\circ} \mathrm{C}$ vacuum-degassed water bath for US scanning. The water bath maintained the body temperature of the rats and provided reliable US transmission. After scanning and a 5minute delay, the rat was euthanized under anesthesia by exsanguination of the inferior vena cava. The trachea was tied off to maintain lung volume, and the lungs were removed for examination and photography using a stereo microscope with a digital camera (Spot Flex; Diagnostic Instruments, Inc, Sterling Heights, MI).

\section{Ultrasound Scanning}

An HDI 5000 diagnostic US machine (Philips Healthcare, Andover, MA) with a CL15-7 linear array was used for scanning, as described previously. ${ }^{9}$ The probe was positioned in partial contact with the right thorax and used to scan the right cranial or middle lobe. The machine was set for real-time imaging with B-mode optimization, a 2-cm depth, 1 focus at a $1-\mathrm{cm}$ depth, and 39 frames per second. An initial MI setting of 0.21 was used to align the probe for a clear view of the lung. The primary variable for the threshold determinations was the on-screen MI setting, with a maximum of 0.9. The US field was characterized previously in the water bath with a calibrated hydrophone (model 805; Sonora Medical Systems, Inc, Longmont, CO). ${ }^{9}$ For this study, the measurements were rechecked and found to be essentially the same. The center frequency was approximately $7.6 \mathrm{MHz}$; the pulse repetition frequency was $10 \mathrm{kHz}$; and the pulse duration was 320 nanoseconds. ${ }^{9}$ The total length of the scan was approximately $2 \mathrm{~cm}$, and the $-6-\mathrm{dB}$ width was $1.0 \mathrm{~mm}$. As before, scanning each rat at a single MI setting was used to assess the scan-response trends and the determination of threshold MIs. The water values for peak rarefactional pressure amplitude were derated by an attenuation coefficient of 
$1.1 \mathrm{~dB} / \mathrm{cm} / \mathrm{MHz}^{32}$ for the approximately 6-mm-thick rat chest wall to obtain $0.6,0.8,1.2,1.6$, and $1.9 \mathrm{MPa}$, for MIs of $0.21,0.37,0.52,0.7$, and 0.9 , respectively. ${ }^{9}$

\section{Experimental Plan}

The probe was aimed to obtain a clear bright image of the lung surface, which appeared as a bright horizontal line in the image with secondary A-lines at greater depths. For a test, the US was quickly raised from an MI of 0.21 to the desired MI setting for 5 minutes of scanning. For the shamexposed animals, the same procedure was followed, except that the scanning at elevated MI settings was omitted. The images were used to obtain estimates of the width of the bright lung surface image, the time to the first clear appearance of a comet tail artifact, and the percentage of the bright lung surface involved in comet tail artifacts at the end of the scanning. The image of the scanned lung area was used to find the length, width, and area of the hemorrhagic region on the lung surface with the aid of image analysis software (Spot version 5.1; Diagnostic Instruments, Inc).

When possible, physiologic data were collected for each rat on the heart rate and percent peripheral capillary oxygen saturation $\left(\mathrm{SP}_{\mathrm{O} 2}\right.$ ) using a pulse oximeter (SurgiVet V3395 TPR; Smiths Medical ASD, Inc). This procedure produced mixed results for both measurements due to difficulty in placing the sensor on the paw and to the upper limit of 350 beats per minute $(\mathrm{bpm})$ for the instrument. To obtain stable values, the sensor was set, and the readings were observed for up to 5 minutes. For the pentobarbital anesthesia, the percent $\mathrm{SP}_{\mathrm{O}_{2}}$ could not be measured due to high heart rates, which were determined from recordings from a laboratory electrocardiographic system (ECGA amplifier; Hugo Sachs Electronik, Harvard Apparatus, March, Germany), digitizer (Powerlab 4/30; ADInstruments, Inc, Colorado Springs, CO), and electrocardiographic analysis software (Chart Pro 5 version 5.5.5; ADInstruments, Inc).

Statistical analysis was performed using SigmaPlot version 11.0 software for Windows (Systat Software, Inc, San Jose, CA). The Mann-Whitney rank sum test was used to compare means of the measured parameters, with statistical significance assumed at $P<.05$. The $z$ test of proportions was used to assess the significance of the proportion of 5 rats that had pulmonary hemorrhage for each group scanned at a specific MI. The proportion test was used to locate the threshold between the lowest MI with significant hemorrhage occurrence and the next lower MI setting. In addition, 2-way ANOVA was used to gauge the influence of the anesthetic methods relative to the MI variation.

\section{Results}

For anesthesia by ketamine alone $(\mathrm{n}=15)$, the heart rate and $\mathrm{SP}_{\mathrm{O} 2}$ averaged $308 \pm 39 \mathrm{bpm}$ and $90 \% \pm 4 \%$, respectively, with no difference between results before and after scanning. For the ketamine plus xylazine tests $(\mathrm{n}=10)$, the heart rate and $\mathrm{SP}_{\mathrm{O} 2}$ were $327 \pm 23 \mathrm{bpm}$ and $92 \% \pm 2 \%$ before addition of xylazine and $264 \pm 18 \mathrm{bpm}$ and $82 \% \pm 4 \%$ 5 minutes after addition of xylazine. The latter results show the possible cardiopulmonary depression induced by the addition of xylazine with a reduced heart rate $(P<.001)$ and $\mathrm{SP}_{\mathrm{O} 2}(P<.001)$. The use of ketamine alone as the anesthetic resulted in much less pulmonary capillary hemorrhage than was seen in the previous study. ${ }^{9}$ Scanning at an MI of 0.37 was omitted because hemorrhage was not seen even at the higher MI of 0.52 . Results are listed in Table 1 for the range of the times to first appearance of comet tail artifacts, for the percentage of bright lung surface images with comet tail artifacts after scanning, for the hemorrhagic areas on the lung, and for the fractions of lungs with positive hemorrhage results. On the basis of the

Table 1. Results for Ketamine Anesthesia

\begin{tabular}{|c|c|c|c|c|c|}
\hline $\begin{array}{l}\text { Group, } \\
\text { MI }\end{array}$ & $\begin{array}{c}\text { PCH Start, } \\
s\end{array}$ & $\begin{array}{c}\text { CTA Width, } \\
\%\end{array}$ & $\begin{array}{c}\text { PCH Area, } \\
\mathrm{mm}^{2}\end{array}$ & $\begin{array}{c}\mathrm{PCH} \\
\text { Fraction }\end{array}$ & $\begin{array}{c}z \text { Test } \\
P\end{array}$ \\
\hline Sham & NA & NA & 0 & $0 / 4$ & NA \\
\hline 0.52 & NA & NA & 0 & $0 / 5$ & NS \\
\hline 0.7 & $210-240$ & $2.6 \pm 4.0$ & $0.1 \pm 0.2$ & $2 / 5$ & NS \\
\hline $0.7-x$ & 300 & 0 & $0.2 \pm 0.2$ & $2 / 5$ & NS \\
\hline $0.7+x$ & 2-60 & $91 \pm 10$ & $7.2 \pm 2.6$ & $5 / 5$ & $<.01$ \\
\hline 0.9 & $10-300$ & $26 \pm 20$ & $1.8 \pm 2.1$ & $5 / 5$ & $<.01$ \\
\hline $0.9+X$ & 5-10 & $94 \pm 6$ & $14.6 \pm 5.0$ & $5 / 5$ & $<.01$ \\
\hline
\end{tabular}

Means \pm SDs include 0 for rats with no discernible hemorrhage, so that the observed sizes in the positive results were approximately given by the means divided by the positive fractions. CTA indicates comet tail artifact; NA, not applicable; NS not significant; PCH, pulmonary capillary hemorrhage; $-X$, test with xylazine added after scanning; +X, test with xylazine added before scanning. 
significant occurrence (5/5) at an MI of 0.9 and the lack of significance $(2 / 5)$ at an MI of 0.7 , the MI threshold was about 0.8 . This finding compares to the loss of significance between MIs of $0.52(4 / 5)$ and $0.37(2 / 5)$ in the previous study, which indicated an MI threshold of about $0.44 .{ }^{9}$ The results with xylazine added before the scanning were comparable to the previous results, but adding the xylazine after scanning did not enhance the hemorrhage above that seen for ketamine alone. A 2-way ANOVA for the 0.7 and 0.9 MIs without and with xylazine showed that both the xylazine and MI had a significant influence on the results even after allowing for the influence of the other variables; however, the interaction effect was only marginally significant $(P=.052)$. The direct comparison for ketamine with xylazine given before or after scanning at an $\mathrm{MI}$ of 0.7 showed a dramatic increase in hemorrhage when xylazine was present during scanning (Table 1), with a significant difference in occurrence $(P=.01)$. Figure 1 compares the stereo microscopic images for the 5 repetitions of each test. Interestingly, the 2 shallow hemorrhagic regions for xylazine addition after scanning did not have any clear indication of comet tail artifacts in the US images, whereas the hemorrhage that penetrated deeper into the lung (darker regions) had comet tail artifacts extending to the bottom of the images.

For pentobarbital anesthesia $(n=30)$, the heart rate averaged $377 \pm 32 \mathrm{bpm}$. The relatively high heart rate precluded $\mathrm{SP}_{\mathrm{O} 2}$ measurement. The use of pentobarbital as the anesthetic resulted in pulmonary capillary hemorrhage that was comparable to that seen in the previous study using ketamine plus xylazine. ${ }^{9}$ Results are listed in Table 2 for the same parameters as in Table 1. On the basis of the significant occurrence $(4 / 5)$ at an $\mathrm{MI}$ of 0.52 and insignificant occurrence $(2 / 5)$ at an $\mathrm{MI}$ of 0.37 , the MI threshold was about 0.44 . This threshold was the same as that found for ketamine plus xylazine in the previous study. ${ }^{9}$ No additional tests were performed with added xylazine for pentobarbital anesthesia because the cardiovascular and pulmonary impact of such a combination could be detrimental. ${ }^{27}$

For isoflurane anesthesia $(\mathrm{n}=17)$, the heart rate and $\mathrm{SP}_{\mathrm{O} 2}$ averaged $343 \pm 11 \mathrm{bpm}$ and $91 \% \pm 2 \%$, respectively, with no observed difference between the results before and after scanning. For the 2 groups with xylazine $(\mathrm{n}=10)$, the heart rate and $\mathrm{SP}_{\mathrm{O} 2}$ were $260 \pm 17 \mathrm{bpm}$ and $90 \% \pm 3 \%$.

Figure 1. Photomicrographs of the right lung cranial lobes from 10 rats scanned at an Ml of 0.7 with ketamine and without (top row) or with (bottom row) added xylazine during scanning (Table 1 ). All of the lobes scanned with added xylazine have substantial pulmonary capillary hemorrhage evident within the linear extent of the scan plane. The lobes scanned without xylazine show no hemorrhage in 3 lobes and small surface evidence of hemorrhage in 2 (black arrows). The lungs had several features that were not due to the US scanning: a fluid-filled region due to impact with a rib (white arrow) and various wrinkles due to slight folding during removal. In addition, a few scanned or sham lungs had very small (possibly 1 alveolus) red spots, which were not scored as pulmonary capillary hemorrhage. Scale bar indicates $5 \mathrm{~mm}$.
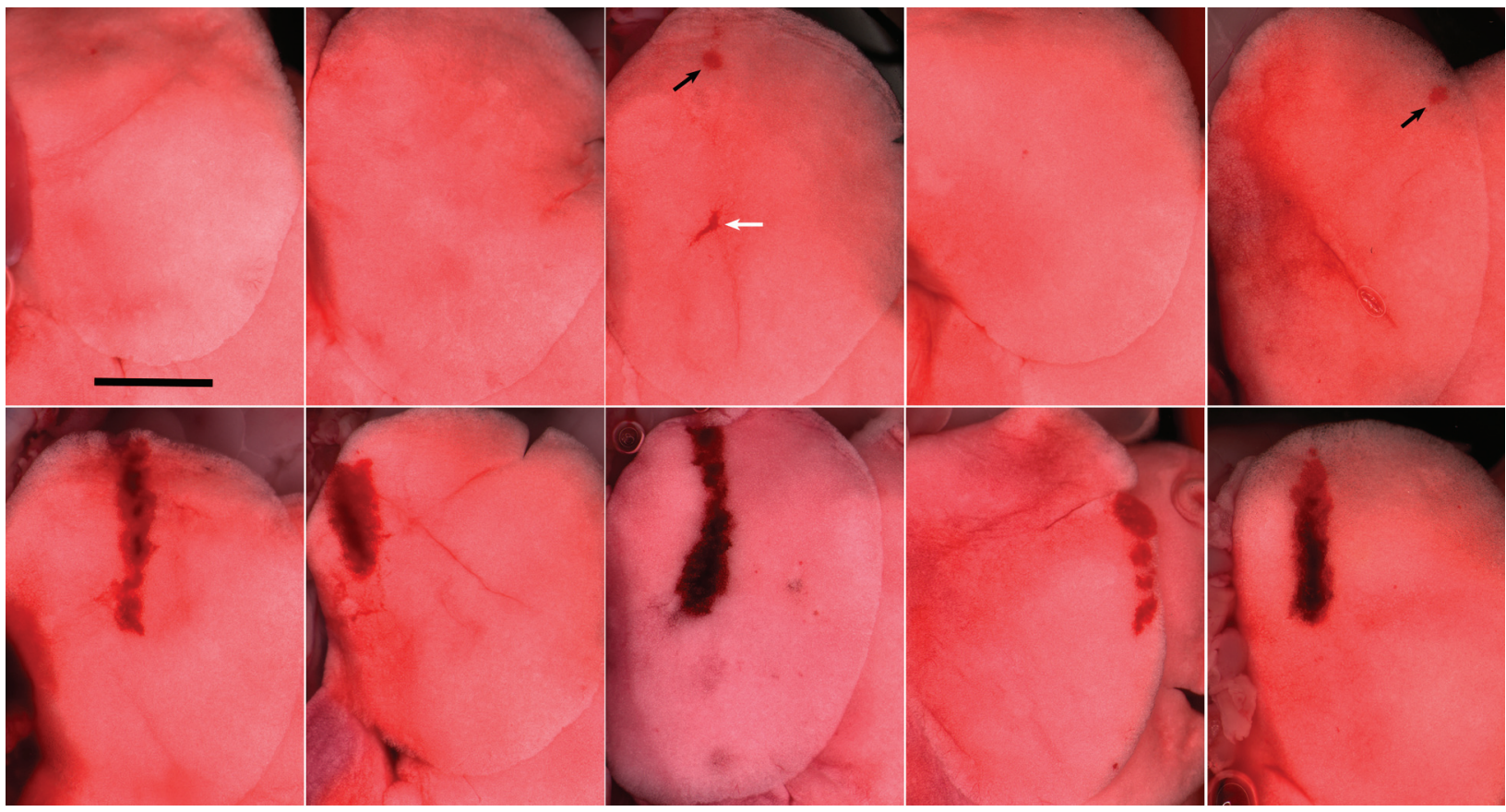
Results are listed in Table 3 for the same parameters as in Tables 1 and 2. No tests were conducted for MIs of 0.27 and 0 (sham) because no effect was found for 5 rats scanned at an MI of 0.37 . On the basis of the significant occurrence $(5 / 5)$ at an MI of 0.9 and insignificant occurrence $(2 / 5)$ at an MI of 0.7 , the MI threshold was about 0.8 . This threshold was the same as that found for ketamine alone (Table $1)$. However, when xylazine was added, pulmonary capillary hemorrhage was found in 5 of 5 rats at an MI of 0.52 $(P=.01)$ but 0 of 5 rats at an MI of 0.37 . This finding indicated a lowering of the threshold for isoflurane plus xylazine to that found for pentobarbital anesthesia and ketamine plus xylazine anesthesia. A 2-way ANOVA for the 0.37 and $0.52 \mathrm{MIs}$ without and with xylazine showed that both the xylazine and MI had significant influence on the results $(P<.01)$, and that there was a significant interaction $(P=.037)$.

The comet tail artifact widths are presented in Figure 2 as the percentage of the lung surface seen as a bright line in the image, shown with the means including all 5 results (ie, 0 for the negative results). The bright line was indicative of perpendicular reflection, which also often generated repeated "A-lines" of multiple reflections at higher depths on the image. The comet tail artifacts developed within this bright-surface image region, which ranged from 7 to $18 \mathrm{~mm}$ in width. The comet tail artifact width correlated well with the actual width of the hemorrhagic area found in the lungs.
This plot shows the variation of pulmonary capillary hemorrhage induced by identical scanning but with different anesthetic techniques, with pentobarbital giving the greatest hemorrhage and ketamine the least. The hemorrhage with pentobarbital was comparable to that found for ketamine with xylazine. ${ }^{9}$ The addition of xylazine to ketamine during scanning raised the result at an MI of 0.7 to $90 \%$ (Table 1), which was higher than the result for pentobarbital. Likewise, the addition of xylazine for isoflurane anesthesia at an MI of 0.52 elevated the comet tail artifact width to $68 \%$ (Table 3 ), which was about the same as for pentobarbital. Interestingly, the low result for isoflurane at an MI of 0.7 was not significantly different from the values obtained with ketamine but was significantly lower than the values obtained with pentobarbital. However, the isoflurane result increased at an MI of 0.9 so that the reverse was true: significantly greater than the ketamine result $(P<.05)$ but not significantly different from the pentobarbital result.

The areas showing pulmonary capillary hemorrhage on the lung surface, which may be a better gauge of the magnitude of the effect than the comet tail artifact, are plotted in Figure 3. The trends were essentially the same as in Figure 2 for comet tail artifacts. For pentobarbital anesthesia, the increases in the areas were more pronounced with increases in the MI than for the comet tail artifact widths. This aspect of the 2-dimensional measurement likely reflects the changes in the width of the ultrasonic beam,

Table 2. Results for Pentobarbital Anesthesia

\begin{tabular}{lccccc}
\hline $\begin{array}{l}\text { Group, } \\
\text { MI }\end{array}$ & $\begin{array}{c}\text { PCH Start, } \\
\text { s }\end{array}$ & $\begin{array}{c}\text { CTA Width, } \\
\%\end{array}$ & $\begin{array}{c}\text { PCH Area, } \\
\mathbf{m m}^{2}\end{array}$ & $\begin{array}{c}\text { PCH } \\
\text { Fraction }\end{array}$ & $\begin{array}{c}\boldsymbol{z} \text { Test } \\
\boldsymbol{P}\end{array}$ \\
\hline Sham & NA & NA & 0 & $0 / 5$ & NA \\
0.27 & NA & NA & 0 & $0 / 5$ & NA \\
0.37 & $7-240$ & $32 \pm 35$ & $0.5 \pm 0.9$ & $2 / 5$ & .11 \\
0.52 & $5-50$ & $65 \pm 40$ & $4.9 \pm 3.3$ & $4 / 5$ & .01 \\
0.7 & $10-90$ & $71 \pm 18$ & $8.3 \pm 4.2$ & $5 / 5$ & $<.01$ \\
0.9 & $1-15$ & $100 \pm 0$ & $21.4 \pm 10.5$ & $5 / 5$ & $<.01$ \\
\hline
\end{tabular}

Notations are as in Table 1.

Table 3. Results for Isoflurane Anesthesia

\begin{tabular}{|c|c|c|c|c|c|}
\hline $\begin{array}{l}\text { Group, } \\
\text { MI }\end{array}$ & $\begin{array}{c}\text { PCH Start, } \\
\text { s }\end{array}$ & $\begin{array}{c}\text { CTA Width, } \\
\%\end{array}$ & $\begin{array}{c}\text { PCH Area, } \\
\mathrm{mm}^{2}\end{array}$ & $\begin{array}{c}\mathrm{PCH} \\
\text { Fraction }\end{array}$ & $\begin{array}{c}z \text { Test } \\
P\end{array}$ \\
\hline 0.37 & NA & NA & NA & $0 / 5$ & NA \\
\hline $0.37+x$ & NA & NA & NA & $0 / 5$ & NA \\
\hline 0.52 & $120-240$ & $6.7 \pm 9.3$ & $1.3 \pm 2.4$ & $2 / 5$ & NS \\
\hline $0.52+X$ & 10-60 & $68 \pm 30$ & $6.4 \pm 4.4$ & $5 / 5$ & $<.01$ \\
\hline 0.7 & $2-20$ & $23 \pm 33$ & $2.6 \pm 3.9$ & $2 / 5$ & NS \\
\hline 0.9 & $0.5-15$ & $90 \pm 14$ & $17.5 \pm 8.6$ & $5 / 5$ & $<.01$ \\
\hline
\end{tabular}

Notations are as in Table 1. 
over which the critical threshold level was exceeded. A 2-way ANOVA for the 0.7 and 0.9 MIs and the 3 anesthetic methods (without xylazine) showed that the effect of the MI on the results was significantly influenced by the drug present $(P=.047)$. At an MI of 0.9 , ketamine was significantly different from pentobarbital and isoflurane anesthesia.

\section{Discussion}

In this study, the influence of different anesthetic techniques on pulmonary capillary hemorrhage induced by diagnostic US was investigated. The hemorrhage was greatest for pentobarbital anesthesia, lower for isoflurane inhalational anesthesia, and lowest for ketamine anesthesia (Figures 2 and 3), with thresholds at MIs of about 0.44, 0.8, and 0.8, respectively. Addition of xylazine produced a substantial increase in hemorrhage and a significant proportion of hemorrhage occurrence for ketamine at an MI of 0.7 (Table 1) and for isoflurane at an MI of 0.52 (Table 3).

These results indicated that the cardiopulmonary effects of xylazine and pentobarbital yield lower thresholds than for ketamine or isoflurane alone by nearly a factor of 2 in MI. The time at which the comet tail artifacts were first seen on the images provides another interesting factor for analysis. The US interaction with the lung surface must begin at the initial pulse. However, the changes in the comet tail artifact start time may be viewed as changes in the time needed to induce substantial pulmonary capillary

Figure 2. Means \pm SEs for the percentages of bright-line lung images which were involved with comet tail artifacts (CTAs) at the end of scanning (Tables 1-3). The occurrence of pulmonary capillary hemorrhage was statistically significant for Mls of 0.5 and higher for pentobarbital but only for an Ml of 0.9 for isoflurane and ketamine-only anesthesia.

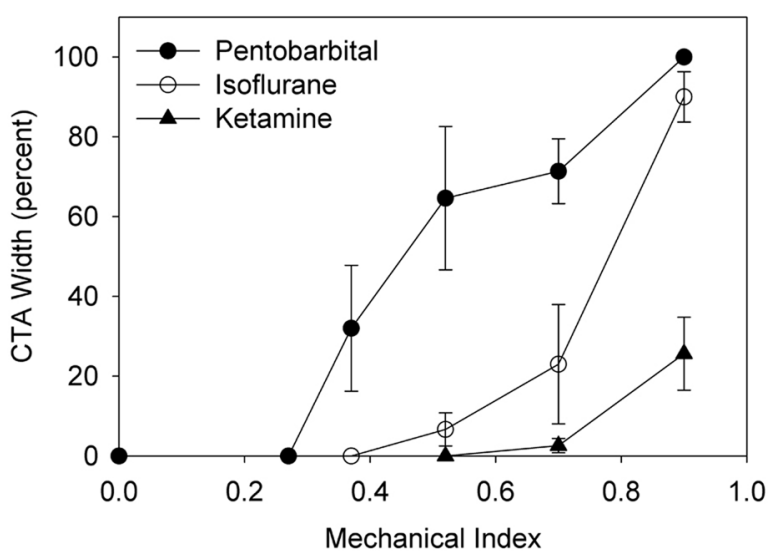

hemorrhage; that is, xylazine and pentobarbital may reduce the time needed (Tables 1-3). If the experimental scan duration were reduced (eg, from 5 minutes to 1 minute), then the 1 of 5 and 2 of 5 results in Tables 1-3 might be eliminated. Unfortunately, the time-to-start parameter was somewhat subjective and variable, and the shallow hemorrhagic areas in Figure 1 did not correspond to any clear comet tail artifact indications. A more definitive appraisal, for example, in terms of a temporal threshold at each MI, would require a different method.

The variation of the sensitivity of the lungs to different anesthetic techniques is likely due to variations in pulmonary physiologic responses. The moderate cardiopulmonary depression caused by xylazine or pentobarbital probably influences the depth of respiration. The degree of lung inflation has been shown to influence the acoustical impedance of the pleural surface, such that low inflation increases the impedance above that of a simple air-water interface. ${ }^{33,34}$ It seems possible that the respiratory depression may therefore result in enhanced energy transmission into the lungs and enhanced interaction with the pulmonary tissue.

The sensitivity to US-induced pulmonary capillary hemorrhage shown for anesthesia with xylazine or pentobarbital is likely also related to the physiologic response of the pulmonary microvasculature. The pulmonary capillary pressure is normally only about 2 to $15 \mathrm{~mm} \mathrm{Hg}$. ${ }^{35}$ Higher pressure values distend the capillaries and apply some level of stress to the capillary border ${ }^{36}$ Pressure values above about $30 \mathrm{~mm} \mathrm{Hg}$ are sufficient to induce edema. ${ }^{37}$

Figure 3. Means \pm SEs for lung surfaces with evident pulmonary capillary hemorrhage for the 3 anesthetics. As in Figure 2, the results were statistically significantly different from shams at Mls of 0.5 and higher for pentobarbital but only for an $\mathrm{Ml}$ of 0.9 for isoflurane and ketamine-only anesthesia.

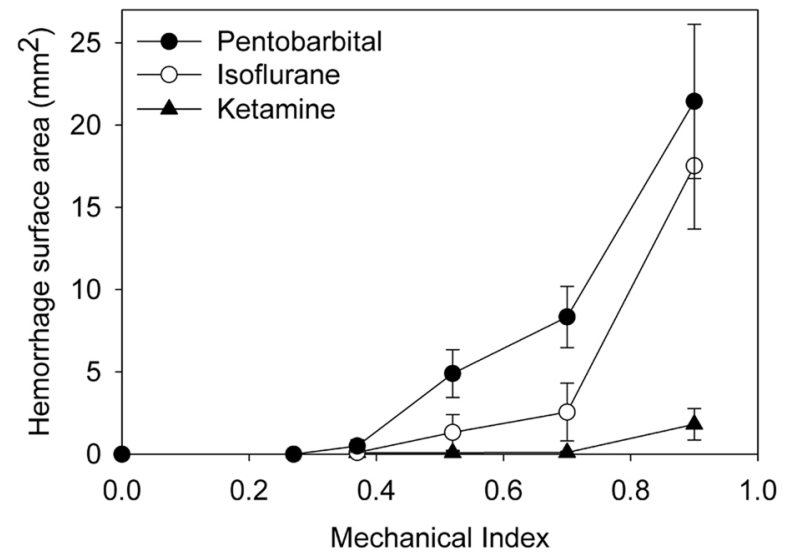


Values higher than 40 to $50 \mathrm{~mm} \mathrm{Hg}$ can cause capillary rupture. ${ }^{35}$ Xylazine is an $\alpha 2$ receptor agonist and affects the pulmonary circulation, increasing the pulmonary capillary pressure. As noted above, relatively high doses of xylazine, in the range of 21 to $45 \mathrm{mg} / \mathrm{kg}$, cause pulmonary edema in a few hours. ${ }^{28,29}$ Studies in sheep show that $\alpha 2$ receptor agonists similar to xylazine produce elevated pulmonary capillary pressures in a dose-dependent manner. ${ }^{38,39}$ Pentobarbital causes cardiopulmonary depression with a relatively high heart rate, which may also lead to pulmonary microvascular perturbation, leading to increased pulmonary capillary hemorrhage. Both xylazine and pentobarbital enhanced neurogenic pulmonary edema in rats. ${ }^{40}$ The filling and distension of the capillaries are likely to reduce the amount of exogenous stress required to rupture the capillaries. Therefore, the additional stress required to cause pulmonary capillary hemorrhage may be reduced by xylazine or pentobarbital. The physiologic responses to these drugs likely prestress the microvasculature and make the capillaries vulnerable to the physical stress generated by the exposure to pulsed US.

Most studies of pulmonary capillary hemorrhage induced by US have used anesthetic techniques including xylazine, which may have resulted in relatively low thresholds. The observation that the milder anesthetic methods with ketamine and isoflurane used here resulted in higher thresholds may have implications for human pulmonary diagnostic US. For example, ketamine alone produces a dissociated state, which is not far from wakefulness. It is entirely possible to extrapolate that awake healthy animals, or patients, may be at relatively low risk of pulmonary capillary hemorrhage induced by pulmonary US. Conversely, pulmonary vulnerability may vary with different drugs and patient conditions. For example, drugs that are $\alpha 2$ receptor agonists such as clonidine (used for hypertension, attention deficit/hyperactivity disorder, and sedation), ${ }^{41}$ may have effects that are similar to those of xylazine. Various patient conditions can also produce pulmonary vasodilation with pulmonary venous hypertension. For example, left heart impairment due to heart disease can lead to increased pulmonary pressures and edema. ${ }^{42}$ Reduced air pressures due to upper airway obstruction or high altitudes ${ }^{43} \mathrm{can}$ also lead to vasodilation and edema. These drug effects and patient conditions may cause a relatively high risk of pulmonary capillary hemorrhage from pulmonary US. Interestingly, the use of xylazine for anesthesia in this and previous pulmonary capillary hemorrhage research may have fortuitously mimicked susceptible physiologic conditions and provided data relevant to high-risk patients. Further research on US-induced pulmonary capillary hemorrhage is needed to clarify the problem of physiologic variability for guiding sonographers in the safe use of pulmonary US for all patient conditions.

\section{References}

1. Child SZ, Hartman CL, Schery LA, Carstensen EL. Lung damage from exposure to pulsed ultrasound. Ultrasound Med Biol 1990; 16:817-825.

2. American Institute of Ultrasound in Medicine. Section 4: bioeffects in tissues with gas bodies. JUltrasound Med 2000; 19:97-108, 154-168.

3. Food and Drug Administration. Information for Manufacturers Seeking Marketing Clearance of Diagnostic Ultrasound Systems and Transducers. Rockville MD: Food and Drug Administration, Center for Devices and Radiological Health; 2008

4. Church CC, O'Brien WD Jr. Evaluation of the threshold for lung hemorrhage by diagnostic ultrasound and a proposed new safety index. Ultrasound Med Biol 2007; 33:810-818.

5. Church CC, Carstensen EL, Nyborg WL, Carson PL, Frizzell LA, Bailey MR. The risk of exposure to diagnostic ultrasound in postnatal subjects: nonthermal mechanisms. J Ultrasound Med 2008; 27:565-592.

6. Tarantal AF, Canfield DR. Ultrasound-induced lung hemorrhage in the monkey. Ultrasound Med Biol 1994; 20:65-72

7. Holland CK, Deng CX, Apfel RE, Alderman JL, Fernandez LA, Taylor KJ. Direct evidence of cavitation in vivo from diagnostic ultrasound. Ultrasound Med Biol 1996; 22:917-925.

8. Meltzer RS, Adsumelli R, Risher WH, et al. Lack of lung hemorrhage in humans after intraoperative transesophageal echocardiography with ultrasound exposure conditions similar to those causing lung hemorrhage in laboratory animals. J Am Soc Echocardiogr 1998; 11:57-60.

9. Miller DL. Induction of pulmonary hemorrhage in rats during diagnostic ultrasound. Ultrasound Med Biol 2012; 38:1476-1482.

10. Sartori S, Tombesi P. Emerging roles for transthoracic ultrasonography in pleuropulmonary pathology. World J Radiol 2010; 2:83-90.

11. Feller-Kopman D. Ultrasound-guided thoracentesis. Chest 2006; 129 : $1709-1714$

12. Hyacinthe AC, Broux C, Francony G, et al. Diagnostic accuracy of ultrasonography in the acute assessment of common thoracic lesions after trauma. Chest 2012; 141:1177-1183.

13. Lichtenstein DA. Ultrasound examination of the lungs in the intensive care unit. Pediatr Crit Care Med 2009; 10:693-698.

14. Stefanidis K, Dimopoulos S, Nanas S. Basic principles and current applications of lung ultrasonography in the intensive care unit. Respirology $2011 ; 16: 249-256$.

15. Zieleskiewicz L, Contargyris C, Brun C, et al. Lung ultrasound predicts interstitial syndrome and hemodynamic profile in parturients with severe preeclampsia. Anesthesiology 2014; 120:906-914.

16. Reissig A, Copetti R, Kroegel C. Current role of emergency ultrasound of the chest. Crit Care Med 2011;39:839-845

17. Koenig SJ, Narasimhan M, Mayo PH. Thoracic ultrasonography for the pulmonary specialist. Chest 2011; 140:1332-1341. 
18. Dalecki D, Child SZ, Raeman CH, Cox C, Penney DP, Carstensen EL. Age dependence of ultrasonically induced lung hemorrhage in mice. Ultrasound Med Biol 1997; 23:767-776.

19. O’Brien WD Jr, Yang Y, Simpson DG. Threshold estimation and superthreshold behavior of ultrasound-induced lung hemorrhage in rats: role of age dependency. Ultrasound Med Biol 2009; 35:129-135.

20. Baggs R, Penney DP, Cox C, et al. Thresholds for ultrasonically induced lung hemorrhage in neonatal swine. Ultrasound Med Biol 1996; 22:119128.

21. O’Brien WD Jr, Simpson DG, Ho MH, Miller RJ, Frizzell LA, Zachary JF. Superthreshold behavior and threshold estimation of ultrasoundinduced lung hemorrhage in pigs: role of age dependency. IEEE Trans Ultrason Ferroelectr Freq Control 2003; 50:153-169.

22. Zachary JF, Sempsrott JM, Frizzell LA, Simpson DG, O’Brien WD Jr. Superthreshold behavior and threshold estimation of ultrasound-induced lung hemorrhage in adult mice and rats. IEEE Trans Ultrason Ferroelectr Freq Control 2001; 48:581-592.

23. O'Brien WD, Simpson DG, Frizzell LA, Zachary JF. Threshold estimates and superthreshold behavior of ultrasound-induced lung hemorrhage in adult rats: role of pulse duration. Ultrasound Med Biol 2003; 29:1625-1634.

24. O’Brien WD Jr, Simpson DG, Frizzell LA, Zachary JF. Superthreshold behavior of ultrasound-induced lung hemorrhage in adult rats: role of pulse repetition frequency and pulse duration.J Ultrasound Med 2006; 25:873-882.

25. Raeman CH, Child SZ, Carstensen EL. Timing of exposures in ultrasonic hemorrhage of murine lung. Ultrasound Med Biol 1993; 19:507-512.

26. Raeman CH, Child SZ, Dalecki D, Cox C, Carstensen EL. Exposure-time dependence of the threshold for ultrasonically induced murine lung hemorrhage. Ultrasound Med Biol 1996; 22:139-141.

27. Flecknel P. Laboratory Animal Anaesthesia. London, England: Academic Press; 2009.

28. Amouzadeh HR, Sangiah S, Qualls CWJr, Cowell RL, Mauromoustakos A. Xylazine-induced pulmonary edema in rats. Toxicol Appl Pharmacol 1991; 108:417-427.

29. Amouzadeh HR, Qualls CW Jr, WyckoffJH III, et al. Biochemical and morphological alterations in xylazine-induced pulmonary edema. Toxicol Pathol 1993; 21:562-571.

30. Miller DL, Suresh MV, Dou C, Yu B, Raghavendran K. Characterization of ultrasound-induced pulmonary capillary hemorrhage in rats. Microvasc Res 2014; 93:42-45.

31. Hedenstierna G. Oxygen and anesthesia: what lung do we deliver to the post-operative ward? Acta Anaesthesiol Scand 2012; 56:675-685.

32. Teotico GA, Miller RJ, Frizzell LA, ZacharyJF, O'Brien WDJr. Attenuation coefficient estimates of mouse and rat chest wall. IEEE Trans Ultrason Ferroelectr Freq Control 2001; 48:593-601.

33. O’Brien WD Jr, KramerJM,Waldrop TG, et al. Ultrasound-induced lung hemorrhage: role of acoustic boundary conditions at the pleural surface. JAcoust Soc Am 2002; 111:1102-1109.

34. Oelze ML, Miller RJ, Blue JP Jr, Zachary JF, O’Brien WD Jr. Estimation of the acoustic impedance of lung versus level of inflation for different species and ages of animals. J Acoust Soc Am 2008; 124:2340-2352.
35. Melby SJ, Moazami N, Damiano RJ. Cardiac surgery. In: Klingensmith ME, Chen LE, Glasgow SC, Goers TA, Melby SJ (eds). The Washington Manual of Surgery. 5th ed. Philadelphia, PA: Lippincott Williams \& Wilkins; 2008:509-528.

36. West JB, Mathieu-Costello O. Structure, strength, failure, and remodeling of the pulmonary blood-gas barrier. Annu Rev Physiol 1999; 61:543-572.

37. Stoelting RK, Hillier SC. Pulmonary circulation. In: Pharmacology and Physiology in Anesthetic Practice. 4th ed. Philadelphia, PA: Lippincott Williams \& Wilkins; 2006:741-748.

38. Celly CS, McDonell WN, Black WD. Cardiopulmonary effects of the alpha2-adrenoceptor agonists medetomidine and ST-91 in anesthetized sheep.J Pharmacol Exp Ther 1999; 289:712-720.

39. Kästner SB, Ohlerth S, Pospischil A, Boller J, Huhtinen MK. Dexmedetomidine-induced pulmonary alterations in sheep. Res Vet Sci2 2007; 83:217226.

40. Leal Filho MB, Morandin RC, de Almeida AR, et al. Importance of anesthesia for the genesis of neurogenic pulmonary edema in spinal cord injury. Neurosci Lett 2005; 373:165-170.

41. van Zwieten PA. Antihypertensive drugs interacting with the sympathetic nervous system and its receptors. In: Antonaccio M (ed). Cardiovascular Pharmacology. 3rd ed. New York, NY: Raven Press; 1990:37-73.

42. Guazzi M, Arena R. Pulmonary hypertension with left-sided heart disease. Nat Rev Cardiol 2010; 7:648-659.

43. WestJB, Colice GL, Lee YJ, et al. Pathogenesis of high-altitude pulmonary oedema: direct evidence of stress failure of pulmonary capillaries. Eur Respir J 1995; 8:523-529. 